\title{
Ultrafast dynamics in monolayer transition metal dichalcogenides: Interplay of dark excitons, phonons, and intervalley exchange
}

\author{
Malte Selig ${ }^{0},{ }^{1}$ Florian Katsch, ${ }^{1}$ Robert Schmidt, ${ }^{2}$ Steffen Michaelis de Vasconcellos, ${ }^{2}$ \\ Rudolf Bratschitsch, ${ }^{2}$ Ermin Malic, ${ }^{3}$ and Andreas Knorr ${ }^{1}$ \\ ${ }^{1}$ Nichtlineare Optik und Quantenelektronik, Institut für Theoretische Physik, Technische Universität Berlin, 10623 Berlin, Germany \\ ${ }^{2}$ Institute of Physics and Center for Nanotechnology, University of Münster, 48149 Münster, Germany \\ ${ }^{3}$ Chalmers University of Technology, Department of Physics, SE-412 96 Gothenburg, Sweden
}

(Received 20 March 2019; revised manuscript received 27 August 2019; published 26 September 2019)

\begin{abstract}
Understanding the ultrafast coupling and relaxation mechanisms between valleys in transition metal dichalcogenide semiconductors is of crucial interest for future valleytronic devices. Recent ultrafast pump-probe experiments showed an unintuitive significant bleaching at the excitonic $B$ transition after optical excitation of the energetically lower excitonic $A$ transition. Here, we present a possible microscopic explanation for this surprising effect. It is based on the joint action of exchange coupling and phonon-mediated thermalization into dark exciton states and does not involve a population of the $B$ exciton. Our work demonstrates how intra- and intervalley coupling on a femtosecond timescale governs the optical valley response of $2 \mathrm{D}$ semiconductors.
\end{abstract}

DOI: 10.1103/PhysRevResearch.1.022007

Introduction. Monolayer transition metal dichalcogenides (TMDCs) gained much attention due to their remarkable and rich intra- and intervalley exciton physics [1-7]. The TMDC electronic bandstructure is illustrated in Fig. 1: the energetically lowest optically addressable excitons are formed from electrons and holes located at the $K$ and $K^{\prime}$ points at the corners of the hexagonal Brillouin zone [8,9]. These transitions exhibit a circular dichroism, i.e., the $K\left(K^{\prime}\right)$ point can be excited with left-handed (right-handed) polarized light $\sigma^{+}\left(\sigma^{-}\right)$allowing to selectively excite the opposite valleys at the corners of the Brillouin zone [10]. On short timescales, polarization $\left(\sigma^{+}, \sigma^{-}\right)$resolved pump-probe signals revealed an ultrafast intervalley coupling finding a sub-picosecond timescale for the intervalley transfer between the pumped and unpumped valleys [11-17].

In this Rapid Communication, we study the ultrafast intervalley transfer and relaxation dynamics including simultaneous intervalley exchange and phonon scattering and discuss their role for experimentally observed bleaching in transient pump-probe spectra: after optical excitation of the $A$ transition, a significant bleaching at the $B$ transition was observed [18-21]. Since the energy of the probed $B$ exciton is hundreds of meV larger [9] compared to the $A$ exciton this type of response comes as a surprise. In particular the helicity resolved data in $\mathrm{WS}_{2}$ show that the bleaching of the $B$ transition occurs not only in the unpumped valley but also within the pumped valley which, without considering further interactions, can only be attributed to an ultrafast spin flip of the electron [21] or to a mixture of $A$ and $B$ exciton states [22]. Interestingly, it

Published by the American Physical Society under the terms of the Creative Commons Attribution 4.0 International license. Further distribution of this work must maintain attribution to the author(s) and the published article's title, journal citation, and DOI. was found that the bleaching of the $B$ exciton in the unpumped valley rises faster compared to the pumped valley after optical excitation of the $A$ transition [19-21].

There are several mechanisms which might explain these results: (a) a spin flip within the pumped valley [25,26] would lead to a population of the opposite-spin conduction band, visible as a $B$ transition bleaching in the same valley [21]. (b) Dexter-like intervalley exciton coupling due to electronic wave-function overlap in $k$ space where an $A$ exciton in the pumped valley couples directly to the $B$ exciton in the unpumped valley, whereas the $B$ exciton in the pumped valley is driven through intervalley exchange coupling between $B$ excitons $[19,20]$. However, so far, only rough estimates for the coupling strength are available $[19,20]$. (c) Beyond intervalley coupling also intravalley exchange coupling leads to an exciton-state mixing between $A$ and $B$ excitons but no polarization selective study of this effect is known yet [22]. The processes (b) and (c) are strongly off-resonant and a careful evaluation of the actual coupling strength via $a b$ initio methods is required. Another possible explanation for the observed $B$ transition bleaching is (d) that the $B$ exciton population is driven through stimulated exciton-exciton scattering [23]. This process is strongly excitation dependent and, therefore, contributes only beyond a strict $\chi^{(3)}$ limit.

All proposed mechanisms (a)-(d) may explain specific features of the polarization resolved ultrafast experiments but so far none of them has explained all related features. Therefore, in this Rapid Communication, we introduce a combined mechanism (e), exhibiting several aspects of the mechanisms (a)-(d), at the same time explaining three very specific signatures, seen in all related experiments: (i) the ultrafast intervalley transfer between the $A$ excitons, (ii) the bleaching of the $B$ transition after excitation of the $A$ exciton, as well as (iii) the temporal ordering of the rise times of the different signals and their temperature dependence. 


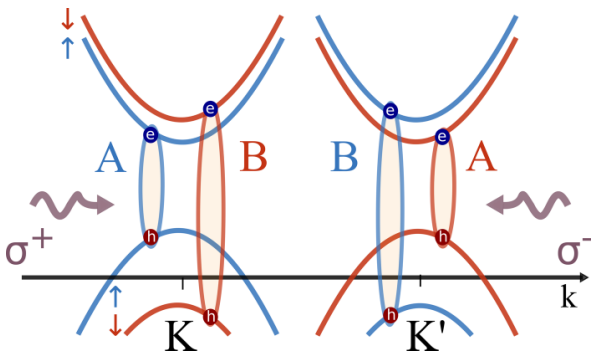

FIG. 1. Schematic illustration of the optical selection rules in $\mathrm{MoSe}_{2}$. A pronounced spin-orbit coupling lifts the degeneracy of the electronic bands where the energetic ordering of the different spin bands (blue, red) is reversed between the $K$ and $K^{\prime}$ points [9,24]. The spin splitting of the conduction band is on the order of few tens of $\mathrm{meV}$ and the splitting of the valence band is on the order of few hundreds of meV. This results in two distinct optical transitions at the $K\left(K^{\prime}\right)$ point where the energetically lower (higher) is called the $A(B)$ transition [9].

Here, we put these observations on a joint consistent theoretical footing: as the dominating mechanism we identify the combined action of the intervalley exchange coupling between the $A$ excitons in both valleys [13,27-32] and phonon mediated scattering to exciton states consisting of electron and hole at opposite $K$ points in the first Brillouin zone [6,33] saturating the $B$ exciton transition. Figure 2 schematically illustrates the proposed mechanism:

First, Fig. 2(a), an $A$ exciton is excited in the $K$ valley with $\sigma^{+}$light. Second, Fig. 2(b), exciton scattering with phonons creates excitons at nonvanishing center of mass momenta, including excitons with electron and hole being located at the same and different high-symmetry point. Third, Fig. 2(c), these excitons, having nonvanishing center of mass momenta initialize the intervalley exchange coupling to the unpumped valley. Finally, Fig. 2(d), the excitons in the unpumped valley scatter to intervalley exciton states. We propose, that these relaxation dynamics is experimentally visible as a bleaching of the $B$ transition on both valleys $\left(\sigma^{+}, \sigma^{-}\right)$, cf. Fig. 2(e), after optical excitation of the $A$ transition in a single valley $\left(\sigma^{+}\right)$.

The bleaching results from Pauli blocking due to the cobosonic nature of excitons [34]. In particular, for the probe of the $B$ transition, we identify signatures from indirect excitons, where the electron of momentum space indirect excitons occupies the conduction band referring to the $B$ transition leading to a bleaching of the latter in the pump-probe signal, cf. Fig. 2(e). In contrast, for the probe of the $A$ transition we identify contributions from the hole of the momentum indirect excitons and from electron and hole of direct excitons, cf. Fig. 2(f). Interestingly, we find that the response at the $B$ transition in the pumped valley $\left(\sigma^{+}\right)$rises slower compared to the unpumped valley $\left(\sigma^{-}\right)$, which results from the stepwise process, cf. Figs. 2(a)-2(d), which is required for the formation of the $B$ exciton $\sigma^{+}$response. This result is in full agreement with recent experiments [13,18,20,21]. We present detailed numerical calculations for the exemplary material $\mathrm{MoSe}_{2}$, but find that our results are also applicable to other TMDC materials.

Theoretical model. We introduce exciton operators $P_{\mathbf{O}}^{\xi_{\mathrm{K}} \xi_{e}}$, where $\xi_{e / h}=\left(i_{e / h}, s_{e / h}\right)$ denotes a compound index consisting
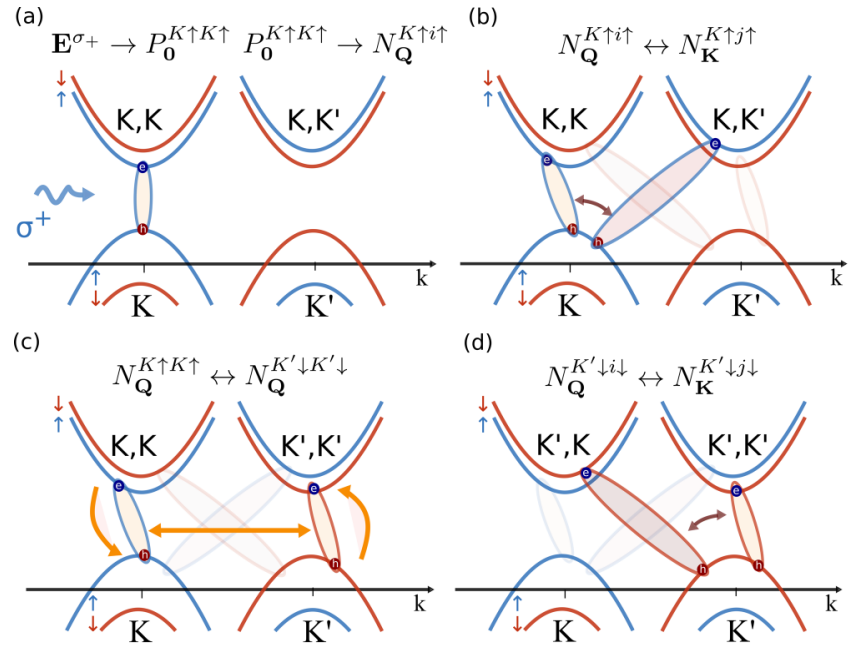

(d)
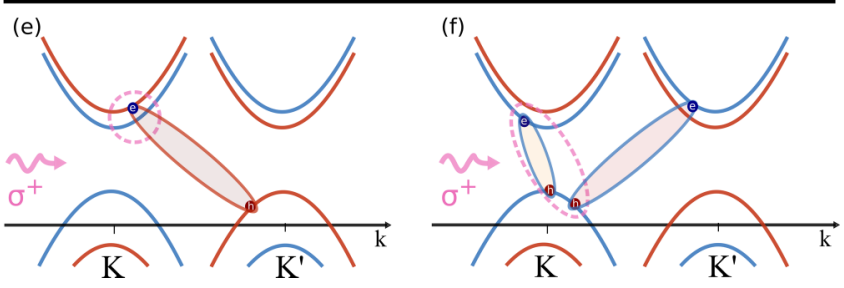

FIG. 2. Schematic illustration of the intervalley exchange in $\mathrm{MoSe}_{2}[(\mathrm{a})-(\mathrm{d})]$ and the pump-probe experiment [(e)-(f)]. (a) Excitation resonantly to the $A$ transition with left handed polarized light $\sigma^{+}$. (b) Exciton phonon scattering mediates the thermalization of excitons: exciton densities in $(K \uparrow, K \uparrow)$ states with nonvanishing center of mass momenta are created. (c) Excitons in $(K \uparrow, K \uparrow)$ and $\left(K^{\prime} \downarrow, K^{\prime} \downarrow\right)$ states are coupled through intervalley exchange coupling. (d) Exciton phonon scattering between excitonic $\downarrow$ states occurs, which also leads to the population of $\left(K^{\prime} \downarrow, K \downarrow\right)$ states. (e) Exemplary for the bleaching of the $B$ transition with $\sigma^{+}$probe pulse, we find contributions from electron of the $\left(K^{\prime} \downarrow, K \downarrow\right)$ exciton. (f) For the bleaching of the $A$ transition with $\sigma^{+}$probe pulse, we find contributions from electron and hole of the $(K \uparrow, K \uparrow)$ exciton and from the hole of the $\left(K \uparrow, K^{\prime} \uparrow\right)$ exciton.

of valley and spin of the electron/hole and the excitonic center of mass momentum $\mathbf{Q}$ and an excitonic Hamiltonian $[34,35]$ to derive the corresponding equations of motion in the Heisenberg picture for expectation values of the excitonic transition $\left\langle P_{\mathbf{Q}}^{\xi_{h} \xi_{e}}\right\rangle$ and the incoherent exciton density $N_{\mathbf{Q}}^{\xi_{h} \xi_{e}}=$ $\delta\left\langle P_{\mathbf{Q}}^{\dagger \xi_{h} \xi_{e}} P_{\mathbf{Q}}^{\xi_{h} \xi_{e}}\right\rangle$, cf. Ref. [36]. The equation of motion for the pumped $A$ exciton transition $\left\langle P_{\mathbf{Q}}^{\xi_{\mathbf{Q}} \xi_{e}}\right\rangle$ in the $K$ valley (in the incoherent $\chi^{(3)}$ limit [37]) reads

$$
\begin{aligned}
i \hbar \partial_{t} P_{\mathbf{0}}^{A \sigma^{+}} & i \hbar \partial_{t} P_{\mathbf{0}}^{K \uparrow K \uparrow}=\left(E_{\mathbf{0}}^{K \uparrow K \uparrow}-i \gamma_{\mathbf{0}}^{K \uparrow K \uparrow}\right) P_{\mathbf{0}}^{K \uparrow K \uparrow}+\mathbf{d}^{K \uparrow} \cdot \mathbf{E}_{\mathrm{pump}} \\
& \times\left(1-\sum_{\mathbf{K} i_{e}} \Xi_{\mathbf{K}}^{K \uparrow,\left(K \uparrow i_{e} \uparrow\right)} N_{\mathbf{K}}^{K \uparrow i_{e} \uparrow}-\Xi_{\mathbf{0}}^{K \uparrow,(K \uparrow K \uparrow \uparrow}\left|P_{\mathbf{0}}^{K \uparrow K \uparrow}\right|^{2}\right) \\
& +\sum_{\mathbf{K}, \xi_{h}, \xi_{e}} W_{\mathbf{K}}^{K \uparrow,\left(\xi_{h}, \xi_{e}\right)}\left(\left|P_{\mathbf{K}}^{\xi_{h}, \xi_{e}}\right|^{2}+N_{\mathbf{K}}^{\xi_{h}, \xi_{e}}\right) P_{\mathbf{0}}^{K \uparrow K \uparrow} .
\end{aligned}
$$




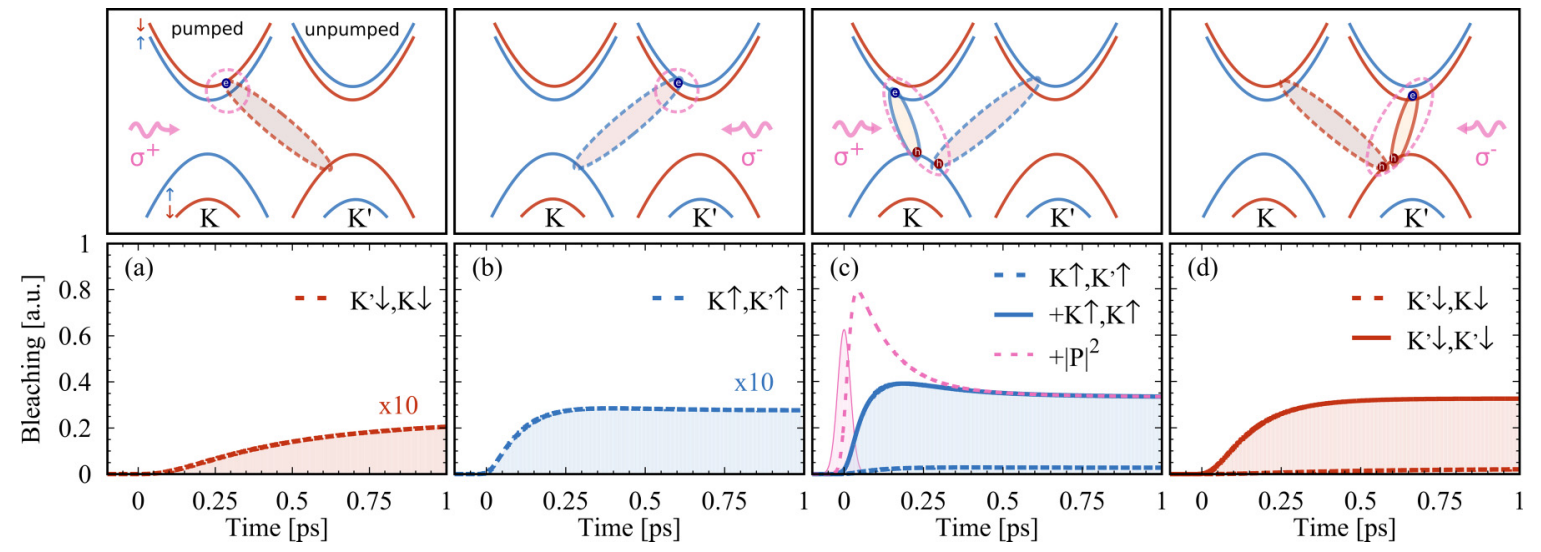

FIG. 3. Bleaching of the pump-probe signal at $77 \mathrm{~K}$ in $\mathrm{MoSe}_{2}$. Contributions from exciton states with spin $\uparrow$ are denoted in blue, spin $\downarrow$ contributions are denoted in red, as in Fig. 2. In the upper panel, we illustrate the excitons leading to the bleaching of the excitonic transition, which is depicted in the lower panel. Contributions from intravalley excitons are illustrated with solid lines, contributions from intervalley excitons are denoted with dashed lines. [(a) and (b)] Pump-probe signal at the $B$ transition, compare Eq. (2), in the pumped (a) and unpumped (b) valley after excitation of the $A$ transition. [(c) and (d)] Pump-probe signal at the $A$ transition, compare equation 1, in the pumped (c) and unpumped (d) valleys after excitation of the $A$ transition. Additionally, we depict the additional bleaching contribution of the optically injected coherence $|P|^{2}$. The pump pulse with a HWHM of $20 \mathrm{fs}$ centered at $t=0 \mathrm{ps}$ is depicted in figure (c) in pink.

For the $B$ transition in the $K$ valley, tested by the probe pulse, the equation of motion reads

$$
\begin{aligned}
i \hbar \partial_{t} P_{\mathbf{0}}^{B \sigma^{+}}= & i \hbar \partial_{t} P_{\mathbf{0}}^{K \downarrow K \downarrow}=\left(E_{\mathbf{0}}^{K \downarrow K \downarrow}-i \gamma_{\mathbf{0}}^{K \downarrow K \downarrow}\right) P_{\mathbf{0}}^{K \downarrow K \downarrow} \\
& +\mathbf{d}^{K \downarrow} \cdot \mathbf{E}_{\text {probe }}\left(1-\sum_{\mathbf{K}} \Xi_{\mathbf{K}}^{K \downarrow,\left(K^{\prime} \downarrow K \downarrow\right)} N_{\mathbf{K}}^{K^{\prime} \downarrow K \downarrow}\right) \\
& +\sum_{\mathbf{K}, \xi_{h}, \xi_{e}} W_{\mathbf{K}}^{K \downarrow,\left(\xi_{h}, \xi_{e}\right)}\left(\left|P_{\mathbf{K}}^{\xi_{h}, \xi_{e}}\right|^{2}+N_{\mathbf{K}}^{\xi_{h} \xi_{e}}\right) P_{\mathbf{0}}^{K \downarrow K \downarrow}
\end{aligned}
$$

The equations of motion for the $A / B$ transition in the $K^{\prime}$ valley can be obtained by exchanging the high-symmetry points $K \leftrightarrow K^{\prime}$ and spins $\uparrow \leftrightarrow \downarrow$. In both equations, the first line accounts for the excitonic dispersion with the excitonic energy $E_{\mathbf{Q}}^{\xi_{h} \xi_{e}}$ and the dephasing, where $\gamma_{\mathbf{0}}^{\xi \xi}$ is determined by radiative decay and exciton phonon coupling $[6,38,39]$. The second line accounts for the optical excitation by the field $\mathbf{E}$ via the dipole moment $\mathbf{d}^{K^{(,)} \uparrow(\downarrow)}$ and for the excitonically modified Pauli blocking of the constituent carriers forming the excitons. The appearing form factors $\Xi_{\mathbf{K}}^{\xi,\left(\xi_{h} \xi_{e}\right)}$ are a consequence of the co-bosonic commutation relation of excitons [34], Eq. (S5). Evaluating the appearing sum over the excitonic valley in Eq. (1) we identify that the bleaching of the $A$ exciton transition results from intravalley $(K, K)$ exciton and the holes of intervalley $\left(K, K^{\prime}\right)$ exciton, cf. Fig. 2(f), whereas for the bleaching of the $B$ transition, we find contributions from the electrons of intervalley $\left(K, K^{\prime}\right)$ excitons only, cf. Fig. 2(e). The third line in Eqs. (1) and (2) schematically describe the energy renormalization due to exciton-exciton interaction with the coupling element $W_{\mathbf{K}}^{\xi,\left(\xi_{h} \xi_{e}\right)}$, Eq. (S6). Note, that we do not calculate the energy renormalization and further many body effects due to Coulomb interaction, which is also required for the interpretation of pump-probe experiments, as shown in Ref. [18], but focus on the bleaching. Equations (1) and (2) together with the equation of motion of the exciton density, Eq. (S13), could be solved iteratively in orders of the exciting optical field to obtain an analytic expression for the third-order susceptibility.
Results. To clarify the temporal pathways of the excitons after optical excitation, we numerically evaluate the coupled dynamics of the excitonic coherence $P_{\mathbf{Q}}^{K \uparrow K \uparrow}$, the exciton densities $N_{\mathbf{Q}}^{\xi_{h} \xi_{e}}$ and the intervalley coherence $\delta\left\langle P_{\mathbf{Q}}^{\dagger K \uparrow K \uparrow} P_{\mathbf{Q}}^{\dagger K^{\prime} \downarrow K^{\prime} \downarrow}\right\rangle$ Eqs. (S10), (S13), and (S14). As it is discussed in Ref. [36], all parameters are determined by microscopic coupling elements. For our numerical evaluation we explicitly include excitons $\left(i_{h} s, i_{e} s\right)$ for both spin bands $s=\uparrow, \downarrow$ but identical spin for electron and hole forming the excitons (optically spin allowed) with electron and hole in the same and in different valleys $i_{h} \neq i_{e}$. For the hole valleys, we include $i_{h}=K, K^{\prime}$ and for the electronic valleys we include $i_{e}=K, K^{\prime}, \Lambda$ (half way between $K$ and $\Gamma$, also referred to as $Q$ or $\Sigma[7,9]), \Lambda^{\prime}$. This way, exploiting Eqs. (1) and (2), we have access to the exciton bleaching

$$
B^{\xi}=\sum_{\mathbf{K}, \xi_{h}, \xi_{e}} \Xi_{\mathbf{K}}^{\xi,\left(\xi_{h}, \xi_{e}\right)} N_{\mathbf{K}}^{\xi_{h}, \xi_{e}}
$$

Figure 3 depicts the polarization $\left(\sigma^{+}, \sigma^{-}\right)$resolved bleaching of the $B / A$ excitons seen by a probe pulse (pink wavy line) as a function of time after optical pump with a $20 \mathrm{fs} \sigma^{+}$pulse resonant to the $A$ transition at an exemplary temperature of $77 \mathrm{~K}$ in $\mathrm{MoSe}_{2}$. On the top, Fig. 3 additionally illustrates the different bleaching contributions of different intervalley $\left(K, K^{\prime}\right)$ and intravalley $(K, K)$ excitons. For all different contributions to the bleaching of $A$ and $B$ excitons we find an ultrafast rise, faster than $1 \mathrm{ps}$.

Figure 3(a) depicts the temporal evolution of the bleaching of the $B$ transition in the pumped valley. As the only contribution we find bleaching from $\left(K^{\prime} \downarrow, K \downarrow\right)$ excitons. The signal rises relatively slow within approximately $700 \mathrm{fs}$, since the formation of these excitons requires a stepwise process as depicted in Figs. 2(a)-2(d): first excitons from the pumped $(K \uparrow, K \uparrow)$ states have to couple to $\left(K^{\prime} \downarrow, K^{\prime} \downarrow\right)$ exciton states through intravalley phonon scattering (or generate $\mathbf{Q} \neq 0$ ) and intervalley exchange, cf. Fig. 2(c), and a subsequent phonon scattering to intervalley ( $\left.K^{\prime} \downarrow, K \downarrow\right)$ states, cf. Fig. 2(d). Such a 


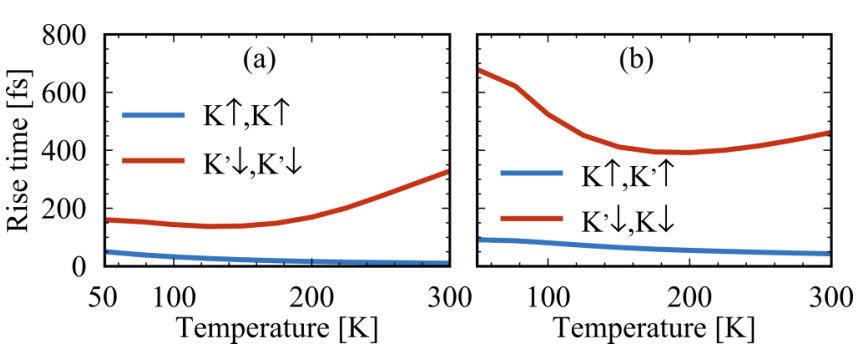

FIG. 4. Extracted density rise times in $\mathrm{MoSe}_{2}$. (a) shows the rise time of the direct exciton densities as a function of temperature and (b) shows the indirect exciton densities.

dynamics is observed as a blocking of the probe pulse and contribute similar to the proposed exciton upconversion or spin flips [21,23].

Figure 3(b) shows the temporal evolution of the bleaching of the $B$ transition in the unpumped valley. The only contribution is the bleaching from $\left(K \uparrow, K^{\prime} \uparrow\right)$ excitons. The signal rises faster compared to the signal in the pumped valley with a time constant of 120 fs, cf. Fig. 3(a), since here the contributing $\left(K \uparrow, K^{\prime} \uparrow\right)$ excitons can be directly formed through intervalley phonon scattering from the optically pumped $(K \uparrow, K \uparrow)$ excitons, cf. Fig. 2(b).

Figure 3(c) depicts the temporal evolution of the bleaching of the $A$ transition in the pumped valley, where beyond the incoherent excitons $N_{\mathbf{Q}}^{\xi_{\mathbf{Q}} \xi_{e}}$, also coherent excitons $\left|P_{\mathbf{Q}}^{K \uparrow K \uparrow}\right|^{2}$ contribute for the resonant probe. As the dominating contribution we identify bleaching from $(K \uparrow, K \uparrow)$ excitons and an additional small contribution from $\left(K \uparrow, K^{\prime} \uparrow\right)$ excitons. The signal rises with the pump pulse, since the ( $K \uparrow, K \uparrow)$ excitons are optically pumped. The additional small contribution from intervalley $\left(K \uparrow, K^{\prime} \uparrow\right)$ excitons is due to phonon mediated thermalization of excitons. Since in $\mathrm{MoSe}_{2}$ these states are located energetically above the bright state by some few $\mathrm{meV}$, their contribution is small at cryogenic temperatures.

Finally Fig. 3(d) depicts the temporal evolution of the bleaching of the $A$ transition in the unpumped valley. We find dominating contributions from $\left(K^{\prime} \downarrow, K^{\prime} \downarrow\right)$ excitons and a small contribution from $\left(K^{\prime} \downarrow, K \downarrow\right)$ excitons. The signal rises relatively fast with a time constant of $200 \mathrm{fs}$, since the $\left(K^{\prime} \downarrow, K^{\prime} \downarrow\right)$ excitons are formed from the pumped ( $\left.K \uparrow, K \uparrow\right)$ excitons through intravalley phonon scattering and intervalley exchange coupling. The small contribution from $\left(K^{\prime} \downarrow, K \downarrow\right)$ excitons is due to the phonon mediated thermalization, as for the pumped valley the $\left(K^{\prime} \downarrow, K \downarrow\right)$ exciton states are located energetically above the $\left(K^{\prime} \downarrow, K^{\prime} \downarrow\right)$ excitons, explaining their small contribution to the signal. All in all, the temporal sequence of the signatures at both $B$ transitions was also observed experimentally recently in $\mathrm{WS}_{2}[20,21]$.

Due to the large energetic separation of $150 \mathrm{meV}$ from the $A$ exciton in $\mathrm{MoSe}_{2}$ [9], we find that excitons involving the electronic $\Lambda$ valley have neglectible occupations and a vanishing influence. For the same reason (180 meV detuning), we neglected the lower split off valence band.

To get a more quantitative analysis with respect to experiments $[13,18-21]$, we extract the rise times of the wavenumber integrated exciton occupations, cf. Fig. 4, by fitting their initial temporal evolution exponentially. For the
( $K \uparrow, K \uparrow)$ exciton density, cf. Fig. 4(a), we find decreasing rise times as a function of temperature (about 50 fs at $50 \mathrm{~K}$ and about $10 \mathrm{fs}$ at room temperature). This can be understood from the fact, that the $(K \uparrow, K \uparrow)$ exciton density is directly formed and the optically pumped excitonic coherence fast converted through intravalley phonon scattering to incoherent excitons [6,33]. For the $\left(K^{\prime} \downarrow, K^{\prime} \downarrow\right)$ density, we find an increase from about $160 \mathrm{fs}$ at $50 \mathrm{~K}$ to about $330 \mathrm{fs}$ at room temperature. Here, several counteracting effects contribute: At elevated temperatures, since the intervalley exchange coupling increases with exciton momentum, a hotter exciton distribution leads to a faster intervalley transfer. Counteracting however is the intervalley coherence damping, Eq. (S8), due to phonon scattering at elevated temperatures. Further at elevated temperatures more excitons occupy the indirect $\left(K \uparrow, K^{\prime} \uparrow\right)$ states, effectively slowing down the intervalley transfer.

In Fig. 4(b), the rise times of the intervalley exciton occupation is depicted. We find the rise time of the $\left(K \uparrow, K^{\prime} \uparrow\right)$ states decreasing from $90 \mathrm{fs}$ at $50 \mathrm{~K}$ to about $40 \mathrm{fs}$ at room temperature, being consistent with the direct formation through phonon scattering from the optically induced exciton coherence. The slowest rise time we find for the $\left(K^{\prime} \downarrow, K \downarrow\right)$ excitons, ranging from about $680 \mathrm{fs}$ at $50 \mathrm{~K}$ to about $460 \mathrm{fs}$ at room temperature. The reason for this comparably slow rise time is that for the formation of these excitons first IEC from the $(K \uparrow, K \uparrow)$ states to $\left(K^{\prime} \downarrow, K^{\prime} \downarrow\right)$ states and after that a phonon mediated scattering from the latter to $\left(K^{\prime} \downarrow, K \downarrow\right)$ states is required. Our calculated timescale is in good agreement with the experimentally measured rise times of the $B$ signal of about $200 \mathrm{fs}$ at $77 \mathrm{~K}$ in $\mathrm{WS}_{2}$ [21]. We are aware that in other TMDCs the excitonic landscape deviates from the situation in $\mathrm{MoSe}_{2}$ [33], and therefore a different temperature dependence can occur. However, we expect, that at least the qualitative behavior including the temporal ordering of the different signals as well as the order of magnitude of the rise times are similar in other materials. Additional to the discussed mechanism also spin-flip processes have been found to contribute to the bleaching of the $B$ transition [21]. In our analysis, we do not observe any excitation power dependence of the rise times, since we restricted our analysis to the low density limit to keep the already high numerical complexity on a moderate level.

Conclusion. The microscopic relaxation dynamics including simultaneous intervalley exchange and intervalley phonon scattering significantly contributes to the theoretical understanding of recent experimental findings, in particular to unintuitive experimental results in helicity-resolved ultrafast pump ( $A$ excitons) probe ( $B$ excitons) experiments. All presented calculations are consistent with recent experimental observation: (i) the fast transfer between the $A$ excitons due to intervalley Coulomb exchange [13], (ii) the bleaching at the $B$ transition after optically exciting the $A$ transition $[18,20]$, and (iii) the faster response at the $B$ transition in the unpumped valley compared to the pumped valley [21]. However, it does not explain the photoluminescence emission from the $B$ exciton under $A$ exciton excitation [23], since no $B$ excitons (involving a hole in the lower valence band) are created.

Acknowledgments. We acknowledge fruitful discussions with Dominik Christiansen (TU Berlin). This work was funded by the Deutsche Forschungsgemeinschaft 
(DFG) - Projektnummer 182087777 - SFB 951 (project B12, M.S., A.K.) and Projektnummer KN 427/11-1 (F.K., R.S., S.M., R.B., A.K.). This project has also received funding from the European Unions Horizon 2020 research and innovation program under Grant Agreement No. 734690
(SONAR, F.K., A.K.). E.M. acknowledges financial support from the European Unions Horizon 2020 research and innovative program under grant agreement No. 785219 (Graphene Flagship) as well as from the Swedish Research Council (VR Project No. 2018-00734).
[1] T. C. Berkelbach, M. S. Hybertsen, and D. R. Reichman, Theory of neutral and charged excitons in monolayer transition metal dichalcogenides, Phys. Rev. B 88, 045318 (2013).

[2] Y. Li et al., Measurement of the optical dielectric function of monolayer transition-metal dichalcogenides: $\mathrm{MoS}_{2}, \mathrm{MoSe}_{2}$, $\mathrm{WS}_{2}$, and $\mathrm{WSe}_{2}$, Phys. Rev. B 90, 205422 (2014).

[3] A. Arora, K. Nogajewski, M. Molas, M. Koperski, and M. Potemski, Exciton band structure in layered $\mathrm{MoSe}_{2}$ : from a monolayer to the bulk limit, Nanoscale 7, 20769 (2015).

[4] D. Y. Qiu, T. Cao, and S. G. Louie, Nonanalyticity, Valley Quantum Phases, and Lightlike Exciton Dispersion in Monolayer Transition Metal Dichalcogenides: Theory and First-Principles Calculations, Phys. Rev. Lett. 115, 176801 (2015).

[5] F. Wu, F. Qu, and A. H. MacDonald, Exciton band structure of monolayer $\mathrm{MoS}_{2}$, Phys. Rev. B 91, 075310 (2015).

[6] M. Selig et al., Excitonic linewidth and coherence lifetime in monolayer transition metal dichalcogenides, Nat. Commun. 7, 13279 (2016).

[7] A. Steinhoff et al., Exciton fission in monolayer transition metal dichalcogenide semiconductors, Nat. Commun. 8, 1166 (2017).

[8] A. Steinhoff, M. Rösner, F. Jahnke, T. O. Wehling, and C. Gies, Influence of excited carriers on the optical and electronic properties of $\mathrm{MoS}_{2}$, Nano Lett. 14, 3743 (2014).

[9] A. Kormanyos et al., k.p theory for two-dimensional transition metal dichalcogenide semiconductors, 2D Materials 2, 022001 (2015).

[10] T. Cao et al., Valley-selective circular dichroism of monolayer molybdenum disulphide, Nat. Commun. 3, 887 (2012).

[11] Q. Wang et al., Valley carrier dynamics in monolayer molybdenum disulfide from helicity-resolved ultrafast pump-probe spectroscopy, ACS Nano 7, 11087 (2013).

[12] S. Dal Conte, F. Bottegoni, E. A. A. Pogna, D. De Fazio, S. Ambrogio, I. Bargigia, C. D'Andrea, A. Lombardo, M. Bruna, F. Ciccacci, A. C. Ferrari, G. Cerullo, and M. Finazzi, Ultrafast valley relaxation dynamics in monolayer $\mathrm{MoS}_{2}$ probed by nonequilibrium optical techniques, Phys. Rev. B 92, 235425 (2015).

[13] R. Schmidt et al., Ultrafast coulomb-induced intervalley coupling in atomically thin $\mathrm{WS}_{2}$, Nano Lett. 16, 2945 (2016).

[14] G. Moody, J. Schaibley, and X. Xu, Exciton dynamics in monolayer transition metal dichalcogenides, J. Opt. Soc. Am. B 33, C39 (2016).

[15] T. Smoleński, M. Goryca, M. Koperski, C. Faugeras, T. Kazimierczuk, A. Bogucki, K. Nogajewski, P. Kossacki, and M. Potemski, Tuning Valley Polarization in a $\mathrm{WSe}_{2}$ Monolayer with a Tiny Magnetic Field, Phys. Rev. X 6, 021024 (2016).

[16] G. Plechinger et al., Trion fine structure and coupled spin-valley dynamics in monolayer tungsten disulfide, Nat. Commun. 7, 12715 (2016).

[17] H. Tornatzky, A.-M. Kaulitz, and J. Maultzsch, Resonance Profiles of Valley Polarization in Single-Layer $\mathrm{MoS}_{2}$ and $\mathrm{MoSe}_{2}$, Phys. Rev. Lett. 121, 167401 (2018).
[18] E. A. A. Pogna et al., Photo-induced bandgap renormalization governs the ultrafast response of single-layer $\mathrm{MoS}_{2}$, ACS Nano 10, 1182 (2016).

[19] I. Bernal-Villamil et al., Exciton broadening and band renormalization due to dexter-like intervalley coupling, 2D Materials 5, 025011 (2018).

[20] G. Berghäuser et al., Inverted valley polarization in optically excited transition metal dichalcogenides, Nat. Commun. 9, 971 (2018).

[21] Z. Wang et al., Intravalley spin-flip relaxation dynamics in single-layer $\mathrm{WS}_{2}$, Nano Lett. 18, 6882 (2018).

[22] Guo Liang et al., Exchange-driven intravalley mixing of excitons in monolayer transition metal dichalcogenides, Nat. Phys. 15, 228 (2019).

[23] M. Manca et al., Enabling valley selective exciton scattering in monolayer $\mathrm{WSe}_{2}$ through upconversion, Nat. Commun. 8, 14927 (2017).

[24] D. Xiao, G.-B. Liu, W. Feng, X. Xu, and W. Yao, Coupled Spin and Valley Physics in Monolayers of $\mathrm{MoS}_{2}$ and Other Group-VI Dichalcogenides, Phys. Rev. Lett. 108, 196802 (2012).

[25] L. Wang and M. Wu, Intrinsic electron spin relaxation due to the D'yakonov-Perel mechanism in monolayer $\mathrm{MoS}_{2}$, Phys. Lett. A 378, 1336 (2014).

[26] L. Wang and M. W. Wu, Electron spin relaxation due to D'yakonov-Perel' and Elliot-Yafet mechanisms in monolayer $\mathrm{MoS}_{2}$ : Role of intravalley and intervalley processes, Phys. Rev. B 89, 115302 (2014).

[27] M. Z. Maialle, E. A. de Andrada e Silva, and L. J. Sham, Exciton spin dynamics in quantum wells, Phys. Rev. B 47, 15776 (1993).

[28] A. Vinattieri, J. Shah, T. C. Damen, D. S. Kim, L. N. Pfeiffer, M. Z. Maialle, and L. J. Sham, Exciton dynamics in GaAs quantum wells under resonant excitation, Phys. Rev. B 50, 10868 (1994).

[29] M. M. Glazov, T. Amand, X. Marie, D. Lagarde, L. Bouet, and B. Urbaszek, Exciton fine structure and spin decoherence in monolayers of transition metal dichalcogenides, Phys. Rev. B 89, 201302 (2014).

[30] T. Yu, and M. W. Wu, Valley depolarization due to intervalley and intravalley electron-hole exchange interactions in monolayer $\mathrm{MoS}_{2}$, Phys. Rev. B 89, 205303 (2014).

[31] H. Dery and Y. Song, Polarization analysis of excitons in monolayer and bilayer transition-metal dichalcogenides, Phys. Rev. B 92, 125431 (2015).

[32] M. Selig et al., Quenching of intervalley exchange coupling in the presence of momentum-dark states in TMDCs, arXiv: 1908.11178.

[33] M. Selig et al., Dark and bright exciton formation, thermalization, and photoluminescence in monolayer transition metal dichalcogenides, 2D Materials 5, 035017 (2018).

[34] F. Katsch, M. Selig, A. Carmele, and A. Knorr, Theory of exciton-exciton interactions in monolayer transition 
metal dichalcogenides, Phys. Status Solidi (b) 255, 1800185 (2018).

[35] A. L. Ivanov and H. Haug, Self-consistent theory of the biexciton optical nonlinearity, Phys. Rev. B 48, 1490 (1993).

[36] See Supplemental Material at http://link.aps.org/supplemental/ 10.1103/PhysRevResearch.1.022007 for detailed information about the exploited theory. Additionally it exhibits the exciton dynamics at $300 \mathrm{~K}$.
[37] H. Haug and S. W. Koch, Quantum Theory of the Optical and Electronic Properties of Semiconductors, 5th ed. (World Scientific, Singapore, 2004).

[38] D. Christiansen et al., Phonon Sidebands in Monolayer Transition Metal Dichalcogenides, Phys. Rev. Lett. 119, 187402 (2017).

[39] A. Raja et al., Enhancement of exciton-phonon scattering from monolayer to bilayer $\mathrm{WS}_{2}$, Nano Lett. 18, 6135 (2018). 